\title{
KINEMATICAL PROPERTIES OF PLANETARY NEBULAE
}

\section{Luciana Bianchi (1), Michael Grewing (2), J.Barnstedt (2),Chr.Diesch(2)}

(1) Osservatorio Astronomico di Torino, Italy

(2) Astronomisches Institut Tuebingen, West Germany

We present newly determined expansion velocities for a number of Planetary Nebulae (PNe) which have been observed with the ESO $1.4 \mathrm{~m}$ CAT and the Coude' Echelle Spectrograph (CES), operated at a r.p. of $10^{5}$, corresponding to a resolution of about $3 \mathrm{~km} / \mathrm{s}$ at $\mathrm{H}_{\alpha}$. Two detector systems have been used: the standard ESO Reticon and a two-dimensional photon-counting imaging detector developed at the A.I.T. (ATT-MCP-Camera).

While for a number of extended objects we obtained several observations at different positions to investigate the detailed kinematical structure (see e.g. Bianchi et al. 1987) we show here only results on the PN expansion velocities obtained from data relative to the centre of each object. The expansion velocities obtained for 17 objects allowed also a statistical interpretation and comparison with current theories of nebular evolution (Bianchi and Falcetta 1987).

In the table below we list the expansion velocity determinations for the objects, obtained independently from the Reticon data and from the AIT-MCP data (given in parenthesis). We point out that for those objects where we obtained spectra with both detectors, the final results from the two independent analyses are in excellent agreement, with the exception of one object.

References.

Bianchi ,L. ,Grewing, M. ,Falcet ta,C. ,Baessgen, M. , 1987: in Proto-planetary Nebulae", Prei te-Martinez ed., Reidel, p.153 Bianchi,L.,Falcetta,C.,1987: in"Mass outflows from stars and galactic nuclei", Bianchi and Gilmozzi eds., Reidel, in press

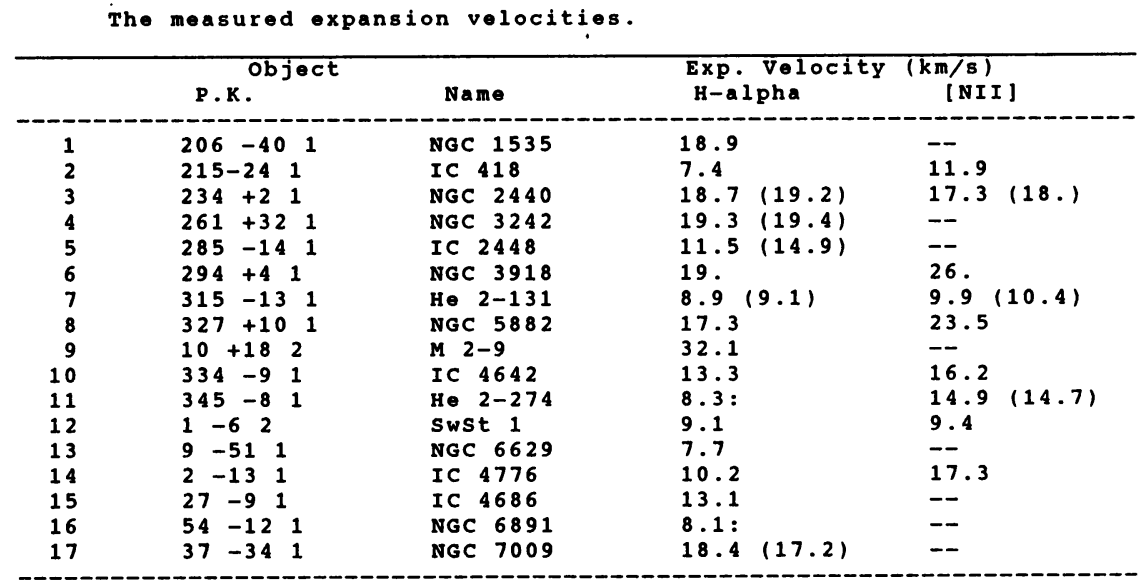

